\title{
A severe genotype with favourable outcome in very long chain acyl-CoA dehydrogenase deficiency
}

\author{
E H Touma, M S Rashed, C Vianey-Saban, A Sakr, P Divry, N Gregersen, B S Andresen
}

\begin{abstract}
A patient with very long chain acyl-CoA dehydrogenase (VLCAD) deficiency is reported. He had a severe neonatal presentation and cardiomyopathy. He was found to be homozygous for a severe mutation with no residual enzyme activity. Tandem mass spectrometry on dried blood spots revealed increased long chain acylcarnitines. VLCAD enzyme activity was severely decreased to $2 \%$ of control levels. Dietary management consisted of skimmed milk supplemented with medium chain triglycerides and L-carnitine. Outcome was good and there was no acute recurrence.

(Arch Dis Child 2001;84:58-60)
\end{abstract}

Keywords: fatty acids; newborn screening; cardiomyopathy; mass spectrometry

Laboratory of Professor Loiselet, Faculty of Medicine, University St Joseph, Damascus Street, PO Box 11-5076, Beirut, Lebanon

E H Touma

Metabolic Screening Laboratory, King Faisal Specialist Hospital, Riyadh, Saudi Arabia M S Rashed

Laboratory of Pediatric

Biochemistry, Hôpital

Debrousse, Lyon,

France

C Vianey-Saban

P Divry

Analytical Testing Laboratory, Beirut, Lebanon

A Sakr

Research Unit for Molecular Medicine and Aarhus University Hospital, Aarhus,

Denmark

N Gregersen

B S Andresen

Correspondence to:

Dr Touma

email: loiselet@dm.net.lb

Accepted 1 June 2000
Very long chain acyl-CoA dehydrogenase (VLCAD) deficiency is a recently identified inborn error of a membrane bound mitochondrial enzyme. ${ }^{1-3}$ We describe a patient with VLCAD deficiency of severe neonatal onset and cardiomyopathy, resulting from a severe mutation type with no residual enzyme activity or "null mutation". Outcome was good with prompt resolution of cardiomyopathy.

\section{Case report}

The patient is the fifth child born to first cousin parents. Two sisters died early in life. The patient developed tachypnoea at 32 hours of life. Laboratory analysis revealed notable acidosis and low blood glucose $(2.8 \mathrm{mmol} / \mathrm{l})$. Other metabolic investigation showed very high serum lactate $(20.2 \mathrm{mmol} / \mathrm{l})$. Organic acid chromatography showed high urinary excretion of some saturated dicarboxylic acids: adipic, suberic, and sebacic acids. The patient was transferred to a university hospital on his fifth day of life and was found to be tachypnoeic on admission with metabolic acidosis ( $\mathrm{pH}$ 7.24). He received intravenous bicarbonate and was started on oral citrate solution and regular feeding. Computed tomography scan of the abdomen revealed an enlarged liver; echocardiography showed mild biventricular hypertrophy with good systolic function. Brain magnetic resonance imaging did not show any abnormal signal. Because of persisting metabolic acidosis and the need for bicarbonate administration, thiamine $150 \mathrm{mg} /$ day, biotin $7.5 \mathrm{mg} /$ day, L-carnitine $250 \mathrm{mg}$ /day, and coenzyme Q $120 \mathrm{mg} /$ day were added to the regimen. Electrospray tandem mass spectrometry (ESI-MSMS) of dried blood spots on filter paper taken three days after L-carnitine treatment identified increased long chain acylcarnitines suggestive of VLCAD deficiency.

At 1.5 months of age the patient was started on low fat milk formula ( $1 \%$ fat in dry powder) supplemented with carbohydrate, medium chain triglyceride (MCT) oil ( $1.5 \mathrm{~g} / \mathrm{kg} /$ day), and L-carnitine $100 \mathrm{mg} / \mathrm{kg} /$ day. At 1 year of age a repeated echocardiogram was normal. Although the patient had some intercurrent infections, his outcome at 3 years of age is still good with normal growth and development and without any recurrence of the metabolic acidosis.

\section{Results}

Acylcarnitine profiling by ESI-MSMS analysis of the patient's blood spot after initiation of treatment with L-carnitine was consistent with VLCAD deficiency (fig 1). Free carnitine was estimated at $62.9 \mu \mathrm{mol} / 1(\mathrm{n}=2$; normal 25-45 $\mu \mathrm{mol} / \mathrm{l})$ on L-carnitine supplementation. The profile showed significantly increased myristoleylcarnitine $(\mathrm{C} 14: 1)$ at $5.6 \mu \mathrm{mol} / 1$ (upper cut off value $0.30 \mu \mathrm{mol} / \mathrm{l}$ ) and increased palmitoylcarnitine $(\mathrm{C} 16)$ at $20.2 \mu \mathrm{mol} / 1$ (cut off 9.30 $\mu \mathrm{M})$ as well as several other long chain acylcarnitines.

On dietary control the determination of tetradecenoic acid $(\mathrm{C} 14: 1, \omega 9)$ in plasma total fatty acids was $17 \mu \mathrm{mol} / 1$ (control <2). ${ }^{4}$ The analysis of plasma acylcarnitines also revealed mild elevation of tetradecenoylcarnitine estimated at $0.7 \mu \mathrm{mol} / 1$ (control $<0.5$ ). Oxidation of palmitic acid by cultured fibroblasts was reduced to $22 \%$ of controls, while VLCAD activity, determined by the dehydrogenation rate of palmitoyl-CoA with electron transferring flavoprotein in cultured fibroblasts, was severely decreased to $0.03 \mathrm{nmol} / \mathrm{min} / \mathrm{mg}$ protein (control: $1.39(0.37) ; \mathrm{n}=28) .^{5}$

Sequence analysis of the entire coding region of the VLCAD gene from a patient as recently published by Andresen and colleagues, ${ }^{6}$ revealed that he was apparently homozygous for a deletion of the nucleotides TG (corresponding to cDNA position 709-710) or GT (corresponding to cDNA position 710-711). This was the only change observed. The mutant allele encodes a VLCAD protein where the reading frame is shifted from the codon encoding cysteine at position 197 to 210 ending with a premature stop codon at codon 211 of the mature protein. To verify that the patient was in fact homozygous for the $2 \mathrm{bp}$ deletion the 


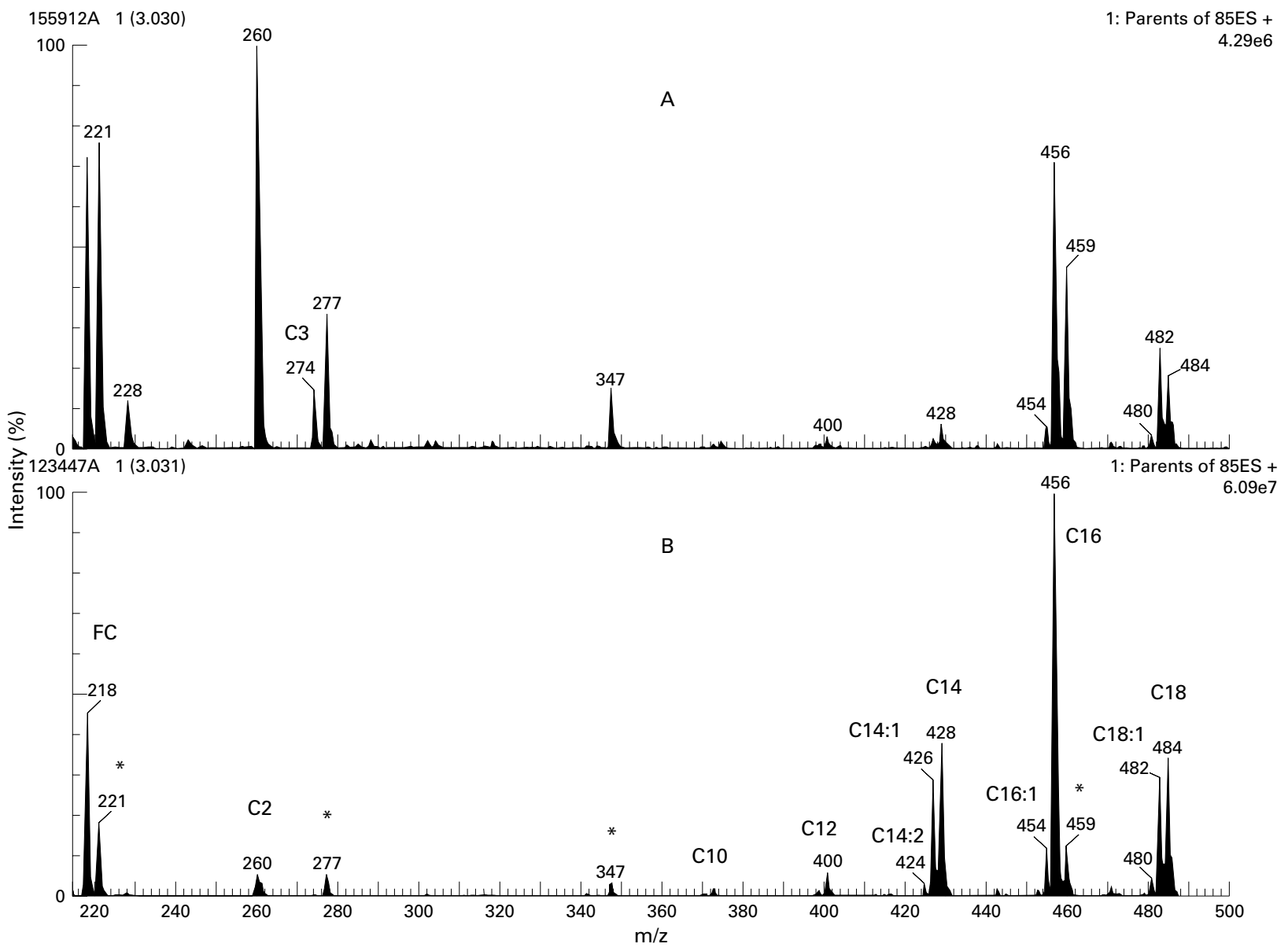

Figure 1 Blood spot free carnitine and acylcarnitine profiles obtained by ESI-MSMS analysis with precursor ion scanning for m/z 85. (A) Control; (B) patient. The peaks in the profile are the molecular ions $\left(M^{+}\right)$of the free carnitine and acylcarnitine butyl esters. Their masses are as follows: free carnitine (FC), 218 ( $d_{3}$ isotope labelled, 221); acetyl (C2), 260; propionyl (C), 274 ( $d_{3}$ isotope labelled, 277); octanoyl (C8), 344 ( $d_{3}$ isotope labelled, 347); decanoyl (C10), 372; dodecanoyl (C12), 400; tetradecanoyl (C14), 428; hexadecanoyl (C16), 456 (d isotope labelled, 459); octadecanoyl (C18), 484.

Their unsaturated analogues appear 2 Da lower in mass. ${ }^{\star}$ Internal standard.

parents were also analysed. Amplification and sequence analysis of exon 8 from the parents, showed that they were both heterozygous for the mutation, thus confirming that the patient is homozygous. The encoded protein would be expected to have no enzyme activity, as vital parts, such as the active site, were lacking. Moreover, analysis of VLCAD mRNA from alleles with premature stop codons, such as the stop codon resulting from the $2 \mathrm{bp}$ deletion described in the present study, have shown that no normal sized mutant VLCAD mRNA can be observed. ${ }^{6}$

\section{Discussion}

Three general forms of clinical presentation in VLCAD deficient patients are known. ${ }^{56}$ The severe childhood form of the disease consists of patients with early onset of symptoms, a very high mortality, or a high number of disease episodes, presence of cardiomyopathy, and siblings who have died. The second group is the mild childhood form and includes patients presenting later in infancy and childhood with a generally milder presentation (fasting induced hypoketotic hypoglycaemia) and fewer episodes of disease precipitation. Cardiomyopathy is rare in this group and mortality much lower. The third group of patients presents in adulthood with an isolated muscular form of the disease (myopathy, rhabdomyolysis, and myoglobinuria). It has recently been shown that patients with the severe childhood form of the disease preferentially have "null" mutations that lead to no residual enzyme activity. ${ }^{6}$

Our patient is considered to have a severe neonatal form with cardiomyopathy and a severe homozygous mutation for a deletion of $2 \mathrm{bp}$ in exon 8 corresponding to cDNA position 710-711. This mutation leaves absolutely no residual enzyme activity and this could explain the fact that the two undiagnosed sisters died at very early age.

In our patient, who has two null mutations, it is striking that all clinical manifestations including cardiomyopathy were completely resolved by appropriate treatment. From this observation it is apparent that patients with two null mutations may avoid disease episodes when fasting is avoided; and that the energy from MCT oil is sufficient to feed the heart and muscle. In conclusion, this study shows that although the heart is nearly obligatory dependent on long chain fatty acid oxidation it is possible to resolve cardiomyopathy in a child with a complete metabolic block of long chain fatty acid oxidation when adequately treated. 
1 Bertrand C, Largilliere C, Zabot MT, Mathieu M, Vianey-Saban C. Very-long-chain acyl-CoA dehydrogenase deficiency: identification of a new inborn error of mitochondrial fatty acid oxidations Acta 1993;1180:327-9.

2 Aoyama T, Uchida Y, Kelley RI, et al. A novel disease with deficiency of mitochondrial very-long-chain acyl-CoA dehydrogenase. Biochem Biophys Res Commun 1993;191:1369-72.

3 Yamaguchi S, Indo Y, Coates PM, Hashimoto T, Tanaka K. Identification of very-long-chain acyl-CoA dehydrogenase deficiency in three patients previously diagnosed with longchain acyl-CoA dehydrogenase deficiency. Pediatr Res 1993;34:111-13.
4 Divry P, Vianey-Saban C, Mathieu M. Determination of total fatty acids in plasma: cis-5-tetradecenoic acid (C14.1.0) in the diagnosis of long-chain fatty acid oxidation defects. F Inherit Metab Dis 1999;22:286-8.

5 Vianey-Saban C, Divry P, Brivet M, et al. Mitochondrial very-long-chain acyl-coenzyme A dehydrogenase deficiency: clinical characteristics and diagnostic considerations in 30 patients. Clin Chim Acta 1998;269:4362 .

6 Andresen BS, Olpin S, Poorthuis BJHM, et al. Clear correlation of genotype with disease phenotype in very-longchain acyl-CoA dehydrogenase deficiency. Am fo Hum Genet 1999;64:479-94.

\section{Polio eradication}

Perhaps it's too early for a full throated crow but we're nearly there, you know: polio should soon go the way of smallpox. In 1999 there were just over 7000 cases of poliomyelitis in the world and during the first half of 2000 there were under 700 cases and 154 isolates of wild poliovirus. The virus is now transmitted in only 30 countries, in South Asia and West and Central Africa (T Jacob John. New England Fournal of Medicine 2000;343:806-7 [editorial]).

In many developing countries antibody responses to oral poliovaccine (OPV), especially to type 1 and 3 virus, are less than in richer countries. Since 1990, infants in Oman have routinely been given five doses of OPV, at birth, at 40 days and at 3, 5, and 7 months. Of 1025 nine month old infants studied in 1992-93 (Roland W Sutter and colleagues. New England Fournal of Medicine 2000;343:767-73) 97\% were seropositive for type 1 poliovirus, $98 \%$ for type 2 , and $88 \%$ for type 3 . They were randomly assigned to one of four supplemental, single dose vaccine options: inactivated poliovirus vaccine (IPV), American-made trivalent OPV, European-made trivalent OPV, or monovalent type 3 OPV. After 30 days none of the OPV options had had any significant effect on type 3 virus antibody titres or seroprevalence but in the infants given IPV, type 3 virus seroprevalence had increased to $97 \%$ and median type 3 antibody titre from 1 in 228 to 1 in 1448. The rapid increase in titre was taken to indicate a secondary immune response.

It is suggested that once wild poliovirus has been eliminated from a country or region, IPV should be used until global eradication is confirmed. A single dose of IPV costs more than a dose of OPV but IPV vaccination is cheaper because fewer doses are needed. In his editorial, $\mathrm{T}$ Jacob John points to the occurrence of vaccine related poliomyelitis and states that polio eradication should mean the elimination of any poliovirus, wild or vaccine strain, from people everywhere. He does not comment directly about the implications for vaccination programmes in the richer countries although he refers, with approval, to experience with IPV in Scandinavia.

ARCHIVIST 